\title{
The role of Runx family in the murine epidermis
}

Eisaku OGAWA ${ }^{1}$, Tomohiro EDAMITSU ${ }^{1}$, Ryuhei OKUYAMA ${ }^{1}$

1. Dermatology, Shinshu university school of medicine, Matsumoto, Nagano, Japan.
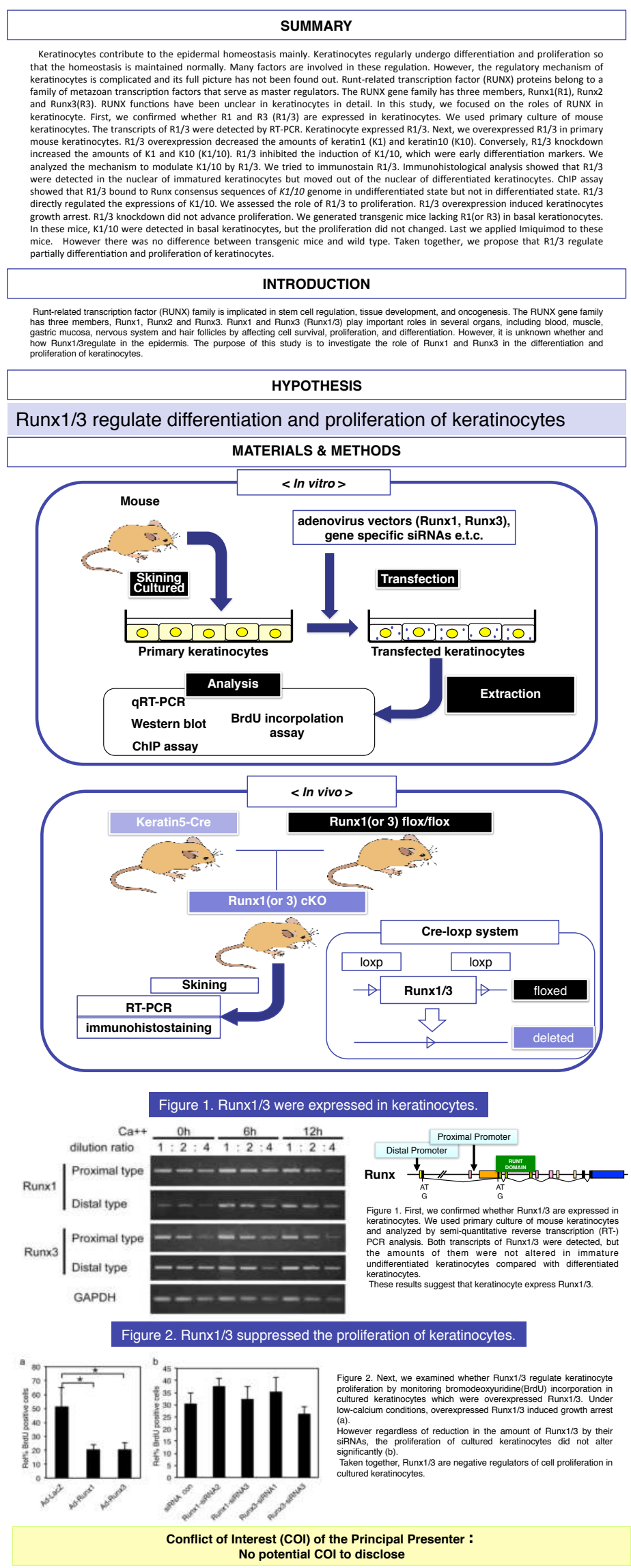
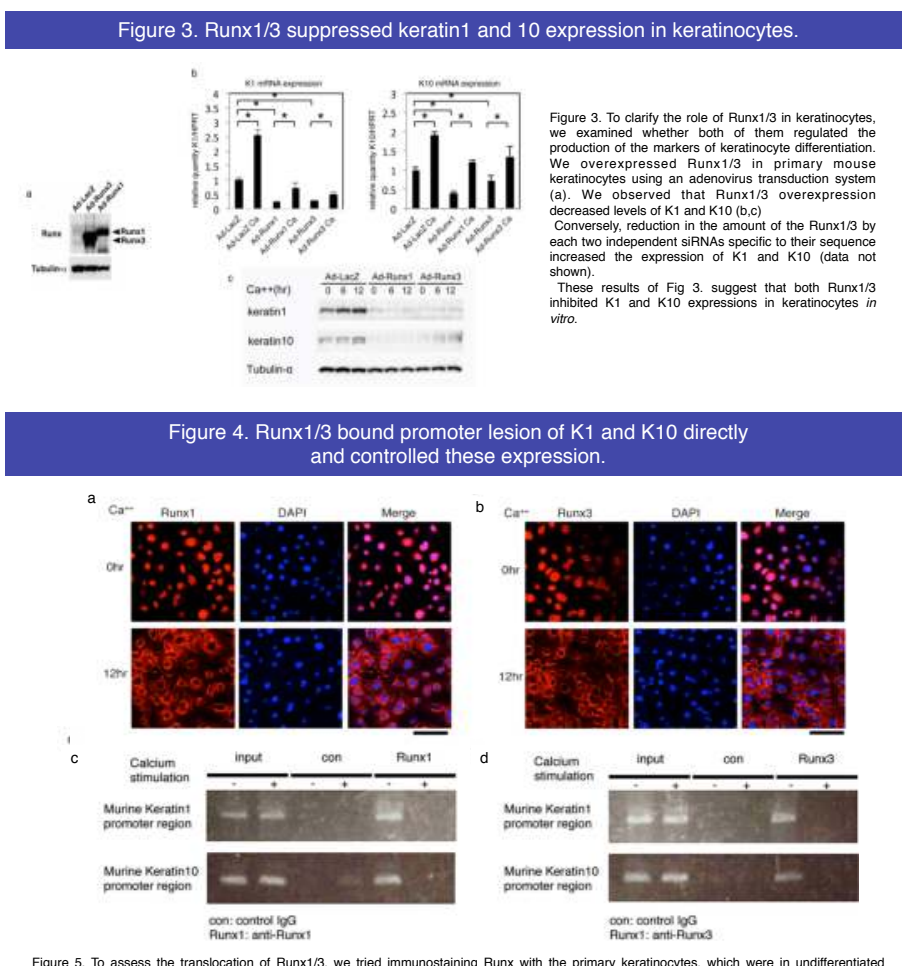

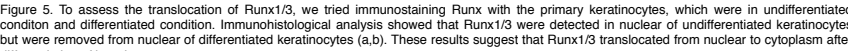

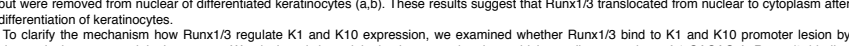
To clarify the mechanism how Runx $1 / 3$ regulate $\mathrm{K} 1$ and $\mathrm{K} 10$ expression, we examined whether Runx $1 / 3$ bind to $\mathrm{K} 1$ and $\mathrm{K} 10$ promoter lesion by
chromatin inmumoprecipitation assay. We designed the original primers each other, which amplicon consists of ' CACAG ", Runx $1 / 3$ binding Under low-calcium condititins, Runx $1 / 3$ bound to $\mathrm{K} 1$ and $\mathrm{K} 10$ promoter lesion (c). However they uncoupled to $\mathrm{K} 1$ and $\mathrm{K} 10$ promoter (d).
Taken together, Runx $1 / 3$ directly regulated the expressions of K1 and K10.

Figure 5. In the skin specific Runx 1 or Runx3 knock-out mice, the expressions of keratin1 and 10 decreased but the proliferation of keratinocyte did not altered.
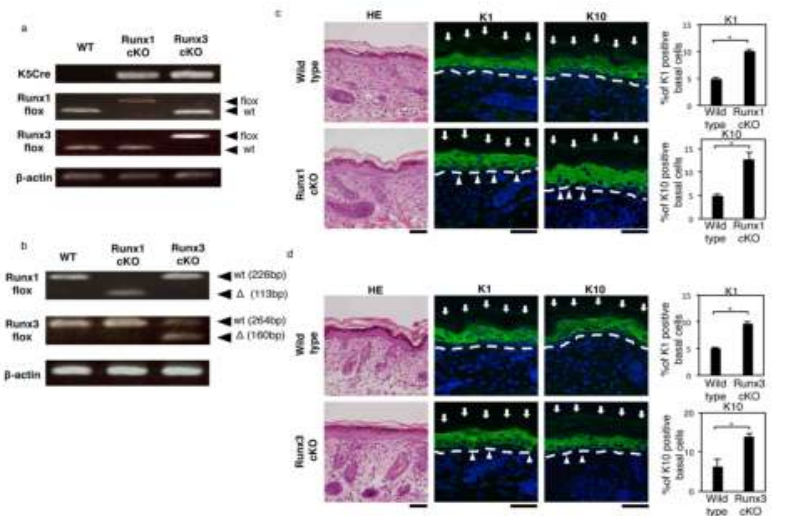

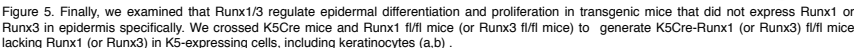

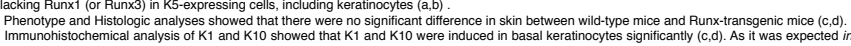

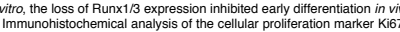

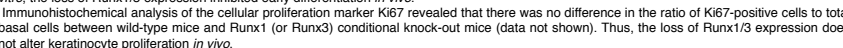

\section{CONCLUSION}

Runx $1 / 3$ inhibits both early differentiation and proliferation of keratinocyte.

Runx $1 / 3$ binds promoter lesion of $\mathrm{K} 1$ and $\mathrm{K} 10$ directly and controls these expression.

\section{ACKNOWLEDGE}

We thank A. Uchiyama for technical assistance; S. Ikawa (Tohoku University) for adenoviral vectors. Supported by the Japan Society for the Promotion of Science (KAKENHI 25461688 and 17 K10236 to E.O.). 